\title{
Publisher's Note: Electrical control of the sign of the $g$ factor in a GaAs hole quantum point contact
} [Phys. Rev. B 94, 041406(R) (2016)]

A. Srinivasan, K. L. Hudson, D. Miserev, L. A. Yeoh, O. Klochan, K. Muraki, Y. Hirayama, O. P. Sushkov, and A. R. Hamilton (Received 18 August 2016; published 24 August 2016)

DOI: 10.1103/PhysRevB.94.079909

This paper was published online on 18 July 2016 with an error in Eq. (3). Equation (3) should read as

$$
g_{x z}=0.39-C_{1} \frac{\left\langle p_{x}^{2}\right\rangle}{\left\langle p_{z}^{2}\right\rangle}-C_{2} \frac{\left\langle p_{y}^{2}\right\rangle}{\left\langle p_{z}^{2}\right\rangle}-C_{3} \frac{\left\langle p_{x}^{2}\right\rangle-\left\langle p_{y}^{2}\right\rangle}{\left\langle p_{z}^{2}\right\rangle}
$$

The equation has been corrected as of 19 August 2016. The equation is incorrect in the printed version of the journal. 